\title{
Prognostic Factors of Initial Chest CT Findings for ICU Admission and Mortality in Patients with COVID-19 Pneumonia
}

\author{
Mohammad Ali Kazemi ${ }^{1,2}$, Hossein Ghanaati ${ }^{2,3}$, Behnaz Moradi ${ }^{2,4}$, Mohammadreza Chavoshi (iD ${ }^{5,}{ }^{*}$, \\ Hassan Hashemi ${ }^{2,3}$, Samira Hemmati ${ }^{1}$, Pouria Rouzrokh ${ }^{6}$, Masoumeh Gity ${ }^{(10}{ }^{2,3}$, Zahra \\ Ahmadinejad ${ }^{7}$ and Hamed Abdollahi ${ }^{8}$ \\ ${ }^{1}$ Department of Radiology, Amiralam Hospital, Tehran University of Medical Sciences, Tehran, Iran \\ ${ }^{2}$ Advanced Diagnostic and Interventional Radiology Research Center (ADIR), Tehran University of Medical Sciences, Tehran, Iran \\ ${ }^{3}$ Department of Radiology, Medical Imaging Center, Imam Khomeini Hospital Complex, Tehran University of Medical Sciences, Tehran, Iran \\ ${ }^{4}$ Department of Radiology, Yas Hospital Complex, Tehran University of Medical Sciences, Tehran, Iran \\ ${ }^{5}$ Department of Radiology, Tehran University of Medical Sciences, Tehran, Iran \\ ${ }^{6}$ Department of Radiology, Shariati Hospital, Tehran University of Medical Sciences, Tehran, Iran \\ ${ }^{7}$ Department of Infectious Diseases, Imam Khomeini Hospital Complex, Tehran University of Medical Sciences, Tehran, Iran \\ ${ }^{8}$ Department of Anesthesiology, Amir Alam Hospital Complexes, Tehran University of Medical Sciences, Tehran, Iran \\ "Corresponding author: Department of Radiology, Shariati Hospital, Tehran University of Medical Sciences, Tehran, Iran. Email: chavoshi.smr@gmail.com
}

Received 2020 June 27; Revised 2020 August 08; Accepted 2020 November 07.

\section{Abstract}

Background: Studies have shown that computed tomography (CT) could be valuable for prognostic issues in COVID-19.

Objectives: To investigate the prognostic factors of early chest CT findings in COVID-19 patients.

Patients and Methods: This retrospective study included 91 patients ( 34 women, and 57 men) of real-time reverse transcription polymerase chain reaction (RT-PCR) positive COVID-19 from three hospitals in Iran between February 25, 2020, to March 15, 2020. Patients were divided into two groups as good prognosis, discharged from the hospital and alive without symptoms (48 patients), and poor prognosis, died or needed ICU care (43 patients). The first CT images of both groups that were obtained during the first 8 days of the disease presentation were evaluated considering the pattern, distribution, and underlying disease. The total CT-score was calculated for each patient. Univariate and multivariate analysis with IBM SPSS Statistics v.26 was used to find the prognostic factors.

Results: There was a significant correlation between poor prognosis and older ages, dyspnea, presence of comorbidities, especially cardiovascular and comorbidities. Considering CT features, peripheral and diffuse distribution, anterior and paracardiac involvement, crazy paving pattern, and pleural effusion were correlated with poor prognosis. There was a correlation between total CT-score and prognosis and an 11.5 score was suggested as a cut-off with $67.4 \%$ sensitivity and $68.7 \%$ specificity in differentiation of poor prognosis patients (patients who needed ICU admission or died). Multivariate analysis revealed that a model consisting of age, male gender, underlying comorbidity, diffused lesions, total CT-score, and dyspnea would predict the prognosis better.

Conclusion: Total chest CT-score and chest CT features can be used as prognostic factors in COVID-19 patients. A multidisciplinary approach would be more accurate in predicting the prognosis.

Keywords: COVID-19, Chest CT, Prognosis, CT-Score

\section{Background}

After two outbreaks of coronavirus by SARS-COV and the Middle East Respiratory Syndrome Coronavirus in 2003 and 2012 respectively, the world faced the third outbreak since December 2019 (1-4). Although the mortality rate was lower than the two previous outbreaks (5), the striking speed of disease spread lead to more death worldwide.

The real-time reverse transcriptase-polymerase chain reaction (RT-PCR) test has been proposed as a specific test for COVID-19, but the sensitivity is as low as $60-70 \%(6,7)$.
Given the limited access for test kits in many countries, there is a high demand for additional diagnostic tests. It is suggested that chest CT scan may have higher sensitivity than RT-PCR for the diagnosis of COVID-19 $(6,7)$. CT scan may help to diagnose the disease in clinically suspicious cases with primary RT-PCR negative results $(8,9)$. According to the stage of the disease, CT scan shows different findings $(10,11)$. The main findings are ground-glass opacity (GGO), consolidation or both in peripheral and lower zones of both lungs (12). However, additional findings 
like crazy-paving pattern, multilobar involvement, and increasing lung consolidations are seen as the disease progresses (7). Some of these findings can determine the severity and extension of the disease $(13,14)$.

Although some laboratory and clinical parameters have been proposed to evaluate the severity of the disease (15-17), limited data is available about the correlation of CT scan findings and disease prognosis. Although CT scan is more useful in the detection or exclusion of viral pneumonia including COVID-19, some CT scan findings may help to investigate the severity of disease and determine the therapeutic approach for the patient.

\section{Objectives}

This study aimed to investigate CT scan findings that could predict mortality or the need for ICU admission in laboratory definite cases of COVID-19.

\section{Patients and Methods}

This study was approved by the Ethics committee of Tehran University of Medical Sciences with the ethical code of IR.TUMS.MEDICINE.REC.1399.095 and written informed consent was waived.

\subsection{Patients}

The data of the patients from three medical centers were evaluated from February 25, 2020, to April 25, 2020, retrospectively. The study population was defined as patients older than 18 years with definite RT-PCR positive diagnosis for COVID-19 who had undergone CT scan examination in less than eight days from the beginning of their symptoms. If the time interval between the RT-PCR assay and the chest CT scan was more than seven days, the patient was excluded at the initial evaluation.

Overall, 104 patients were enrolled into the study; of whom 28 had expired (group A), 16 had ICU admission and subsequent recovery (group B), and 60 patients had no need for ICU admission or outpatient treatment (group C). Follow-up evaluation was done by telephone interview for all discharged patients. Their health status was checked by a medical doctor. All of the discharged patients were alive until May 20, 2020. Twelve patients from group C had missing contact data and were excluded due to loss of follow-up. Another case from group A was also excluded as a synchronous massive pulmonary thromboembolism was found on her CT scan that could have led to her death. Finally, the total number of 91 patients entered the study. Clinical symptoms and history of previous diseases were documented for all patients. Comorbidities were evaluated as cardiovascular disease, pulmonary disease, and other diseases. Diabetes mellitus and hypertension cases were included in the cardiovascular group if history of cardiac complication was positive. Smoking history was asked from the patients and if related pulmonary complications had occurred, the history was considered positive.

\subsection{CT Scan Protocol}

Non-enhanced chest CT scan images were obtained in the supine position, using CT scan systems (SOMATOM Emotion 16 scanner; Siemens). To minimize motion artifacts, CT scan images were acquired during a single inspiratory breath-hold. For minimizing patient radiation exposure, we used following acquisition parameters: tube voltage $=80-110 \mathrm{kVp}$, effective current 60-80 mA, pitch $=1-1.5$, matrix $=512 \times 512$, slice thickness $=5 \mathrm{~mm}$ (reconstructed slice thickness $=1.5 \mathrm{~mm}$ ), and pulmonary U90S kernel. The reconstructed images were sent to the picture archiving and communication system (PACS). The low dose CT scan protocol was recommended by the Iranian Society of Radiology COVID-19 Consultant Group (ISRCC) and did not make any problem in image interpretations (18).

\subsection{Imaging Interpretation}

All chest CT scans were reviewed by two radiologists concurrently with both lung (width, $1500 \mathrm{HU}$; level, -700 $\mathrm{HU}$ ) and mediastinal (width, $350 \mathrm{HU}$; level, $40 \mathrm{HU}$ ) windows. After the final agreement, the prepared checklist was filled. During the review, both radiologists were blinded to the patent's information and outcome. The CT scan evaluation was performed in five major fields: morphology, distribution, CT scan involvement score, associated pulmonary lesions, and mediastinal findings. Morphology features included ground-glass opacity (GGO), consolidation, mixed type with $>50 \%$ GGO, mixed type with $>50 \%$ consolidation, crazy-paving pattern (a combination of GGO with superimposed interlobular and intralobular septal thickening), reversed halo sign, nodular pattern, intralesional bronchial distortion, and linear opacity. Four main distribution patterns were evaluated: peripheral (pleural based or/and pleural sparing), central (patchy opacities that extended to the lung hila and showed lobar bronchial contact without obvious peribroncho-vascular appearance), peri-broncho-vascular (involvement with the disease and not edema) and diffuse (randomly central and peripheral lesions). We expected edema as fine smooth interstitial peribronchovascular 
thickening usually with other imaging supporting findings. Also, the involvement of anterior areas (the involvement of anterior one-fourth of lung periphery in both upper and lower areas), and paracardiac areas (more than 2 $\mathrm{cm}$ in contact with pericardium at lingula and right middle lobe) were evaluated. All lung lobes were visually evaluated for CT scan involvement scores. Each lobe received 0 (non-involvement), 1 (less than 5\% involvement), 2 (5-25\% involvement), 3 (26-49\% involvement), 4 (50-75\% involvement), and 5 (>75\% involvements) (13). A total CT-score was recorded with a range of 0-25. Furthermore, a mean lung score was calculated for each lung by dividing the sum of individual lobar scores of each lung by 3 in the right and 2 in the left lungs. Underlying pulmonary disease as bronchiectasis, emphysema, fibrosis or mass was recorded. The mediastinal setting was assessed for lymphadenopathy, pulmonary artery enlargement, pleural effusion, and pericardial effusion.

\subsection{Statistical Analysis}

IBM SPSS Statistics v.25 (Armonk, NY: IBM Corp) was used for statistical analysis. To determine the prognosis of the patients, groups A and B from above were considered together as the "poor-prognosis" group and group $\mathrm{C}$ was considered as the "good-prognosis" group. Frequencies and descriptive statistics were then calculated for all variables. To determine the relationship between independent (all demographic, clinical and imaging features) and dependent (prognosis) variables, Pearson's chi-square test and the Mann-Whitney U test were used where appropriate. The relationship between the total CT-score and the prognosis was determined by both univariate and multivariate binary logistic regression (non-stepwise) tests. For the latter, a combination of other clinically and statistically significant variables was also considered as inputs along with the total CT-score. Receiver operating characteristic (ROC) curves were plotted for both models and cut-offs with the best trade-off for sensitivity and specificity were proposed to predict the output. The Spearman rank-order correlation test was used to determine the relationship between the total CT-score and the number of days it took from the beginning of the symptoms to the day of performing the CT scan (hereafter called the CT scan day). The relationship between the mean CT-score of each lung and the ipsilateral presence of the pleural effusion was assessed using the Man-Whitney-U test. P values less than 0.05 were considered significant.

\section{Results}

Ninety-one [57 (62.6\%) men and 34 (37.4\%) women] patients were included in the study. The average age of patients was 58.04 years $(S D=16.5)$ and they were admitted to the hospital for a variation of 1 to 18 days (mean $=5.90$, $\mathrm{SD}=4.21) .48$ (52.8\%) patients did not need ICU care and survived, 16 (17.6\%) were admitted to the ICU and survived, 11 (12.0\%) were admitted to the ICU and died, and 16 (17.6\%) died without being admitted to the ICU. Overall, the "goodprognosis" group consisted of 48 (52.7\%) and the "poorprognosis" group consisted of 43 cases (47.3\%). Only eight (8.8\%) cases had normal CT scans and 83 (91.2\%) cases had either ground-glass opacity, consolidations or both (Figure 1). Among the clinical findings, the most common was fever with 81 (89.0\%) of affected cases. Forty-two (46.2\%) of patients had no comorbidities while $18(19.8 \%)$ had cardiovascular, 11 (12.1\%) had pulmonary, eight (8.8\%) had diabetes mellitus (DM), and 12 (13.2\%) had other comorbidities. CT scan and clinical findings/conditions are summarized in Tables 1 and 2.

\begin{tabular}{|c|c|}
\hline Finding & Frequency, No. (\%) \\
\hline Fever & $81(89.0)$ \\
\hline Cough & $67(73.6)$ \\
\hline GI symptoms & $2(2.2)$ \\
\hline Dyspnea & $40(44.0)$ \\
\hline \multicolumn{2}{|l|}{ Underlying comorbidity } \\
\hline Cardiac & $18(19.8)$ \\
\hline Pulmonary & $11(12.1)$ \\
\hline Diabetes Mellitus & $8(8.8)$ \\
\hline Others & $12(13.2)$ \\
\hline ICU admission & $27(29.7)$ \\
\hline Death & $27(29.7)$ \\
\hline \multicolumn{2}{|l|}{ Prognosis } \\
\hline Good $^{\mathrm{a}}$ & $48(52.7)$ \\
\hline $\mathrm{Bad}^{\mathrm{b}}$ & $43(47.3)$ \\
\hline
\end{tabular}

An increase in age and the male gender was associated with poor prognosis. Having underlying comorbidity was also associated with poor-prognosis; $72 \%$ of cases with cardiovascular, $90.9 \%$ of cases with pulmonary, $50 \%$ of cases with DM, and $41.7 \%$ of cases with other comorbidities had poor-prognosis. No significant association was detected between fever, cough or diarrhea and the prognosis, yet 


\begin{tabular}{|c|c|c|}
\hline CT-Scan findings & & Frequencies, No. (\%) \\
\hline & Main CT findings & \\
\hline Normal CT scan & & $8(8.8)$ \\
\hline \multicolumn{3}{|l|}{ Ground glass opacities / Consolidation } \\
\hline Pure GGO & & $10(11.0)$ \\
\hline $\mathrm{GGO}>50 \%$ & & $43(47.3)$ \\
\hline Pure consolidation & & $3(3.3)$ \\
\hline Consolidation $>50 \%$ & & $24(26.4)$ \\
\hline Crazy paving & & $7(7.7)$ \\
\hline Reversed halo sign & & $21(23.1)$ \\
\hline Nodular pattern & & $2(2.2)$ \\
\hline Intralesional bronchial distortion & & $30(33.0)$ \\
\hline Linear opacities & & $44(48.3)$ \\
\hline \multicolumn{3}{|c|}{ Lesion distribution } \\
\hline \multicolumn{3}{|l|}{ Peripheral distribution } \\
\hline Pleural based & & $19(20.9)$ \\
\hline Pleural sparing & & $11(12.1)$ \\
\hline Pleural based and pleural sparing & & $53(58.2)$ \\
\hline Central distribution & & $52(57.15)$ \\
\hline Peribronchovascular distribution & & $20(22.0)$ \\
\hline Diffuse distribution & & $12(13.2)$ \\
\hline Anterior distribution & & $60(65.9)$ \\
\hline Paracardiac distribution & & $30(33.0)$ \\
\hline \multicolumn{3}{|c|}{ Additional findings } \\
\hline Associated bronchiectasis & & $1(1.1)$ \\
\hline Associated emphysema & & $4(4.4)$ \\
\hline Associated fibrosis & & 0 \\
\hline Associated mass & & 0 \\
\hline Lymphadenopathy & & $9(9.9)$ \\
\hline Pulmonary artery enlargement $(>30 \mathrm{~mm})$ & & $14(15.4)$ \\
\hline \multicolumn{3}{|l|}{ Pleural effusion } \\
\hline Trace & & $49(53.8)$ \\
\hline Mild & & $8(8.8)$ \\
\hline \multicolumn{3}{|l|}{ Side of pleural effusion } \\
\hline Right & & $21(23.1)$ \\
\hline Left & & $3(3.3)$ \\
\hline Bilateral & & $33(36.3)$ \\
\hline
\end{tabular}

Abbreviation: GGO: ground glass opacity. 

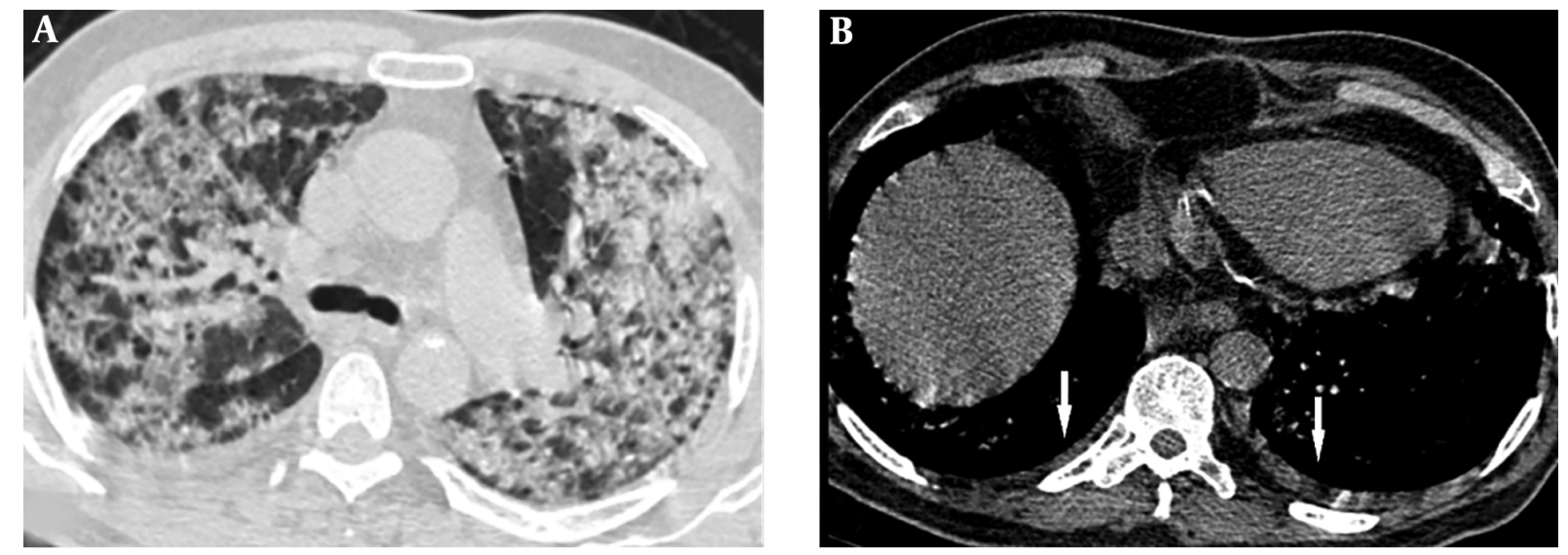

Figure 1. A 68-year-old man who presented with dyspnea 4 days before. A, Axial thin-section unenhanced CT scan shows bilateral diffuse consolidation and ground glass opacities. B, Axial thin-section unenhanced CT scan shows bilateral mild pleural effusion (arrows). The patient was admitted to the ICU 10 days after this CT scan.

dyspnea was associated with poor prognosis. Among CT scan findings, the crazy-paving pattern, peripheral distribution, diffuse distribution, anterior distribution, paracardiac distribution, lymphadenopathy, main pulmonary artery dilation (above $30 \mathrm{~mm}$ ), and pleural effusion were associated with poor prognosis (Figures 2-5). On the other hand, the reversed halo sign was associated with a better prognosis. Test types and the test results of statistically significant associations between patient prognosis and demographic, clinical and CT scan findings are summarized in Table 3. No case of pericardial effusion was seen in this study.

The univariate binary logistic regression model was able to predict the prognosis by the total CT-score $\left(\mathrm{X}^{2}(1)=\right.$ 15.02, $\mathrm{P}<0.001$ ). The model explained $21.8 \%$ (Nagelkerke $\mathrm{R}_{2}$ ) of the variance in prognosis and correctly predicted $68.1 \%$ of cases. Increasing the total CT-score was associated with an increase in the likelihood of poor prognosis (Odds ratio $[\mathrm{OR}]=1.17$ ). The ROC curve for this univariate model is plotted (Figure 6). The AUC for the model is 0.73 $(\mathrm{P}<0.001)$ and by choosing a cut-off of 11.5 for the total CT-score, the resulting sensitivity and specificity would be $67.4 \%$ and $68.7 \%$ respectively. A multivariate binary logistic regression model was also developed including total CTscore, male gender, age, underlying pulmonary or cardiovascular comorbidity, dyspnea, and diffuse distribution of CT scan findings as input variables $\left(\mathrm{X}^{2}(8)=21.65, \mathrm{P}=0.006\right)$. Table 4 shows the statistical significance and ORs for each variable in the latter model. Except for underlying comorbidity, all other input variables significantly contribute to the model. The model explained 64.4\% (Nagelkerke $\mathrm{R}_{2}$ ) of the variance in prognosis and correctly predicted $86.8 \%$ of cases. The ROC curve for this univariate model is plotted (Figure 7). The AUC for the model is $0.94(\mathrm{P}<0.001)$ and by choosing a cut-off of 0.61 for the model output, the resulting sensitivity and specificity would be $81.4 \%$ and $79.0 \%$ respectively.

An increase in the total CT-score had a weak correlation with an increased CT scan day (Spearman's rank two-tailed Correlation, $\mathrm{rho}=0.26, \mathrm{P}=0.023)$, indicating that as the disease progresses in the first week of symptoms, pulmonary involvement in CT becomes more severe. Also, the mean of CT-score for lobes of each lung (mean CT-score) was statistically different between the patients with or without ipsilat-

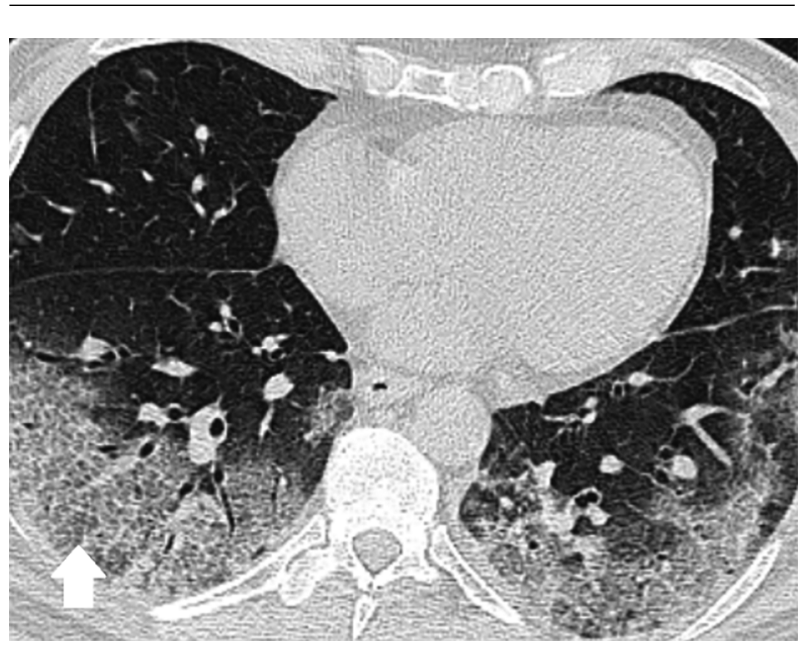

Figure 2. A 55-year-old man who presented with fever and cough 8 days before. Axial thin-section unenhanced CT scan shows peripheral opacities with crazy paving appearance more prominent on the right side. The patient was admitted to the ICU 4 days after this CT scan. 


\begin{tabular}{|c|c|c|c|c|}
\hline Independent variable & Statistical test used & Test results & Good-prognosis & Poor-prognosis \\
\hline Age & Man-Whitney U & $\mathrm{U}=410, \mathrm{P}<0.001$ & Median: 51 & Median: 68 \\
\hline Gender & Chi-square & $\mathrm{X}^{2}(1)=4.83, \mathrm{P}=0.028, \mathrm{Phi}=0.23$ & & \\
\hline Male & & & $43.9 \%$ & $56.1 \%$ \\
\hline Female & & & $67.6 \%$ & $32.4 \%$ \\
\hline Underlying comorbidity & Chi-square & $X^{2}(4)=20.56, P<0.001, P h i=0.47$ & & \\
\hline Cardiac & & & $28.0 \%$ & $72.0 \%$ \\
\hline Pulmonary & & & $9.1 \%$ & $90.9 \%$ \\
\hline Diabetes mellitus & & & $50.0 \%$ & $50.0 \%$ \\
\hline Others & & & $58.3 \%$ & $41.7 \%$ \\
\hline Hospital admit duration, days & Man-Whitney U & $\mathrm{U}=793, \mathrm{P}=0.056$ & Median: 4 & Median: 6 \\
\hline Dyspnea & Chi-square & $X^{2}(1)=11.73, P=0.001 ; P h i=0.359$ & & \\
\hline No & & & $68.6 \%$ & $31.4 \%$ \\
\hline Yes & & & $32.5 \%$ & $67.5 \%$ \\
\hline Crazy-paving & Chi-square & $\mathrm{X}^{2}(1)=4.50, \mathrm{P}=0.034, \mathrm{Phi}=0.22$ & & \\
\hline No & & & $56.0 \%$ & 44.0 \\
\hline Yes & & & $14.3 \%$ & 85.7 \\
\hline Reverse halo sign (RHS) & Chi-square & $\mathrm{X}^{2}(1)=3.82, \mathrm{P}=0.051, \mathrm{Phi}=-0.20$ & & \\
\hline No & & & $47.1 \%$ & 52.9 \\
\hline Yes & & & $71.4 \%$ & 28.6 \\
\hline Peripheral distribution & Chi-square & $\mathrm{X}^{2}(1)=7.8, \mathrm{P}=0.005, \mathrm{Phi}=0.29$ & & \\
\hline No & & & $100 \%$ & $0 \%$ \\
\hline Yes & & & $48.2 \%$ & $51.8 \%$ \\
\hline Diffuse distribution & Chi-square & $X^{2}(1)=10.94, P=0.001, P h i=0.34$ & & \\
\hline No & & & $59.5 \%$ & $40.5 \%$ \\
\hline Yes & & & $8.3 \%$ & $91.7 \%$ \\
\hline Anterior distribution & Chi-square & $\mathrm{X}^{2}(1)=6.26, \mathrm{P}=0.01, \mathrm{Phi}=0.26$ & & \\
\hline No & & & $71 \%$ & $29.0 \%$ \\
\hline Yes & & & $43.3 \%$ & $56.7 \%$ \\
\hline Paracardiac distribution & Chi-square & $X^{2}(1)=6.76, P=0.009, P h i=0.27$ & & \\
\hline No & & & $62.3 \%$ & $37.7 \%$ \\
\hline Yes & & & $33.3 \%$ & $66.7 \%$ \\
\hline Lymphadenopathy & Chi-square & $\mathrm{X}^{2}(1)=3.88 \mathrm{P}=0.049, \mathrm{Phi}=0.20$ & & \\
\hline No & & & $56.1 \%$ & $43.9 \%$ \\
\hline Yes & & & $22.2 \%$ & 77.8 \\
\hline Pulmonary artery dilation & Chi-square & $\mathrm{X}^{2}(1)=3.73 \mathrm{P}=0.053, \mathrm{Phi}=0.20$ & & \\
\hline No & & & $57.1 \%$ & $42.9 \%$ \\
\hline Yes & & & $28.6 \%$ & $71.4 \%$ \\
\hline Pleural effusion & Chi-square & $X^{2}(1)=10.20, P=0.006$, Phi $=0.33$ & & \\
\hline No & & & $70.6 \%$ & $29.4 \%$ \\
\hline Trace & & & $46.9 \%$ & $53.1 \%$ \\
\hline Mild & & & $12.5 \%$ & $87.5 \%$ \\
\hline
\end{tabular}



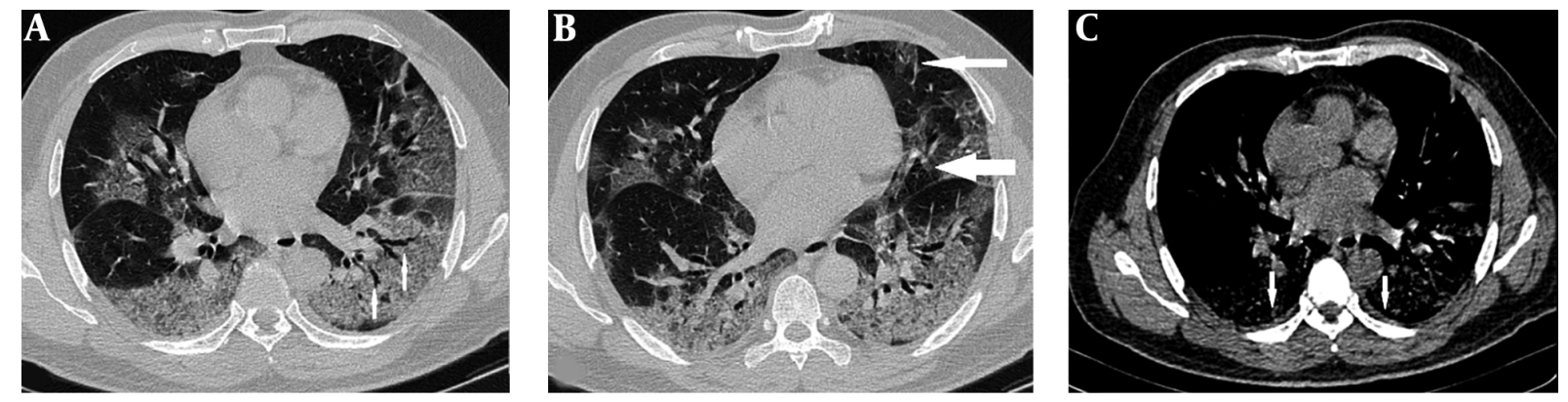

Figure 3. A 59-year-old man who presented with cough, fever and dyspnea 7 days before. A, Axial thin-section unenhanced CT scan shows bilateral peripheral ground-glass opacity (GGO) with intralesional bronchial distortion. B, Axial thin-section unenhanced CT scan with paracardiac (wide arrow) and anterior (narrow arrow) extension of the opacities. This density gradient raises concerns about acute respiratory distress syndrome (ARDS) formation. C, Axial thin-section unenhanced CT scan shows bilateral trace pleural effusion. The patient was admitted to the ICU one day later.

\begin{tabular}{|c|c|c|c|c|}
\hline Independent variable & Significance & Odds ratio & 95\% Confidence interval lower & 95\% Confidence interval upper \\
\hline Total CT-score & 0.050 & 1.17 & 1.00 & 1.38 \\
\hline Male gender & 0.004 & 0.08 & 0.01 & 0.46 \\
\hline Age & 0.003 & 1.08 & 1.02 & 1.14 \\
\hline Comorbidity (cardiovascular and pulmonary) & 0.177 & 2.93 & 0.61 & 13.99 \\
\hline Dyspnea & 0.038 & 3.90 & 1.07 & 14.16 \\
\hline Diffuse distribution & 0.038 & 17.27 & 1.17 & 254.30 \\
\hline Constant & 0.001 & 0.001 & & \\
\hline
\end{tabular}

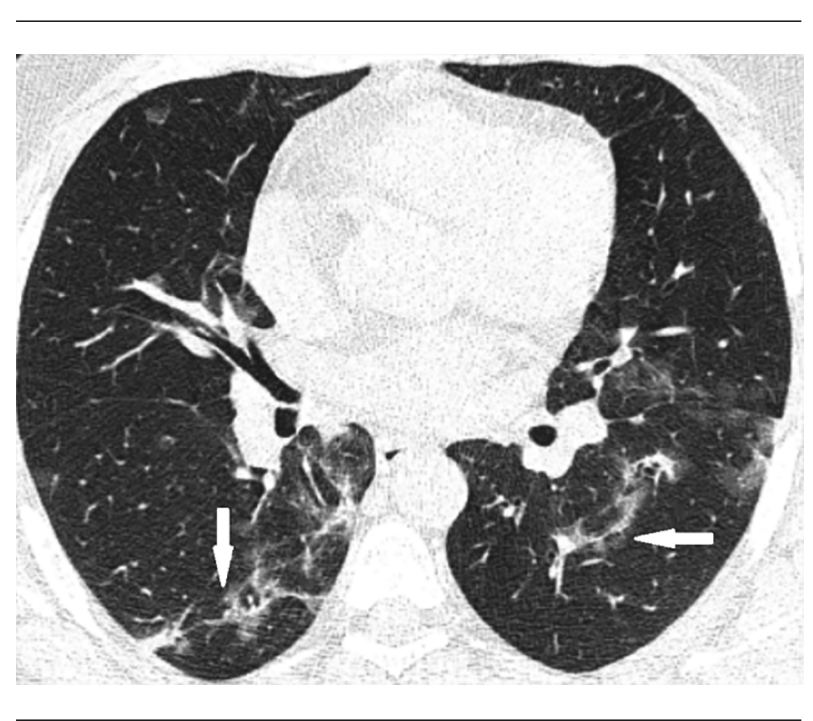

Figure 4. A 40-year-old man who presented with history of fever and cough 7 days before. Axial thin-section unenhanced CT scan shows bilateral peripheral linear opacities (arrows). The patient was treated on an outpatient basis.

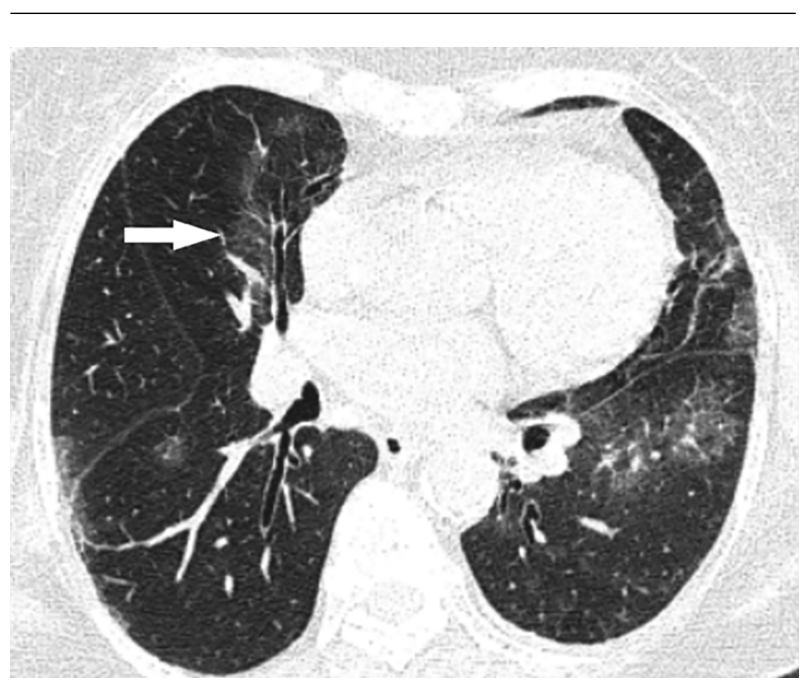

Figure 5. A 87-year-old woman who presented with history of cough and dyspnea 6 days before. Axial thin-section unenhanced CT scan shows peripheral ground glass opacities with paracardiac and anterior extension (arrow). Unfortunately, the patient died 12 days after this exam. 


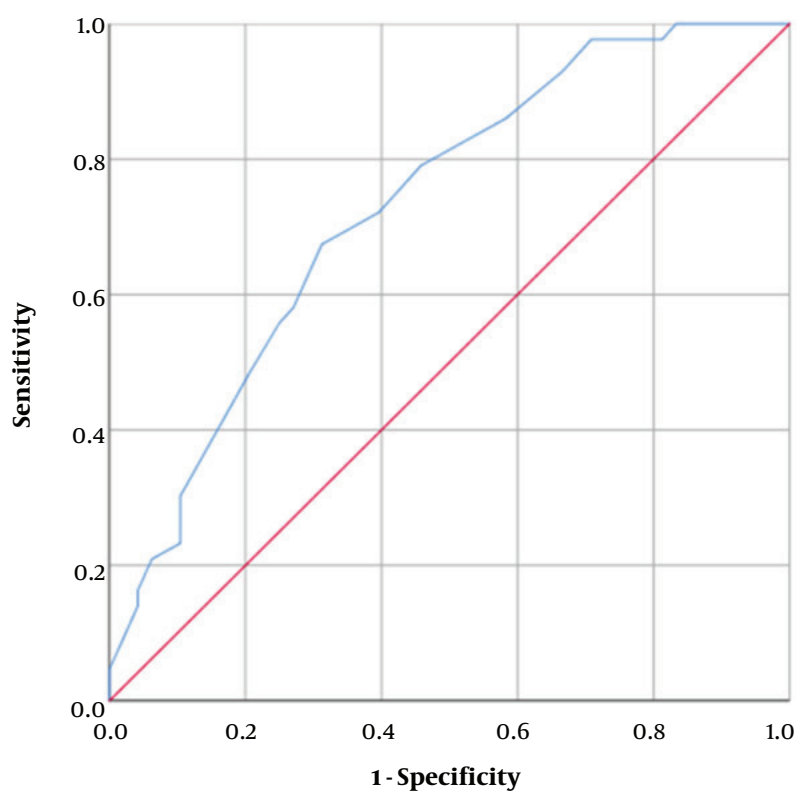

Figure 6. Receiver operating characteristic (ROC) curve for a univariate model to predict patients' prognosis based on their Total CT-score.

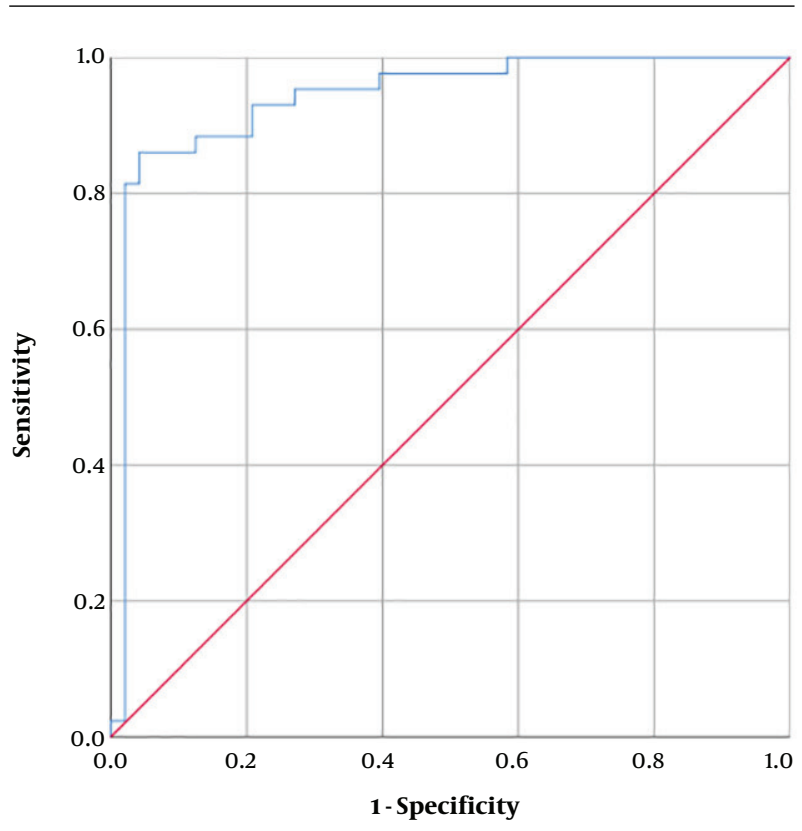

Figure 7. Receiver operating characteristic (ROC) curve for a multivariate model to predict patients' prognosis based on Total CT-score, gender, age, underlying pulmonary or cardiac comorbidity, dyspnea, and diffuse distribution of CT scan findings.

eral pleural effusion (Man-Whitney $\mathrm{U}$ test, $\mathrm{P}<0.001)$. The median and mean CT-score was $1.33(\mathrm{n}=34)$ and $2.66(\mathrm{n}=54)$ in patients with and without right lung pleural effusion, respectively. The same measure was $1.50(n=34)$ and 3.00 $(\mathrm{n}=36)$ in patients with and without left lung pleural effusion, respectively.

Seventy-nine patients (86.8\%) had CT-scores higher than 0 in both lungs, and 12 (13.2\%) patients had at least one lung with CT-score $=0$.

\section{Discussion}

From the beginning day of the COVID-19 epidemic, the diagnosis of the disease became a major challenge. RT-PCR was used as the specific but not sensitive enough test. Further studies showed that the sensitivity and specificity of CT scan are totally acceptable even in comparison with RT$\operatorname{PCR}(6,9)$. This led to a dramatic increase in the number of CT scans in suspicious COVID-19 cases. Although CT scan has a remarkable role in the diagnosis of COVID-19, there is still a dilemma about its role in predicting disease severity and prognosis; so, we comprehensively studied the radiographic features of 93 COVID-19 patients to investigate the prognostic factors of chest CT scan.

Similar to other coronaviruses including SARS, the COVID-19 mostly causes constitutional symptoms including fever, cough, dyspnea, and sometimes gastrointestinal (GI) symptoms $(19,20)$. Like previous studies, fever and cough are the most common symptoms in our patients with $89 \%$ and $74 \%$ prevalence, respectively. However, the only symptom that was correlated with the patient's prognosis was dyspnea. Compatible with previous studies, we found that age and underlying comorbidity (mainly pulmonary and cardiac) has a significant role in prognosis and elderly people with these comorbidities face more severe disease and worse prognosis $(13,19,20)$. Also, we found that the prognosis in men is worse than women proposing the role of gender which needs more evaluations.

Major CT scan findings in our study were consistent with previous reports (10-14). Mixed GGO and consolidation were the most common pattern (73.7\%). GGO was predominantly seen in $47.3 \%$ and consolidation was predominantly seen in $26.4 \%$, followed by exclusively GGO (11\%) and consolidation only (3.3\%). Peripheral distribution was seen in $91.2 \%$, in which $58 \%$ of them showed mixed peripheral and central distribution. Bilateral involvement was seen in $86.8 \%$ of the patients and $8.8 \%$ of them had normal CT scan. Compatible with previous reports, diffuse involvement of the lung was significantly more common in the poor prognosis group in our study $(13,14)$. However, we also found that anterior and paracardiac involvements were related to poor prognosis. In patients with anterior lung involvement superimposed on posterior lesions and presence of 
density gradient we must be worried about progression to acute respiratory distress syndrome (ARDS). Although ARDS is a clinical diagnosis, but classic appearance of acute ARDS in CT scan is anterior-posterior density gradient with dense consolidation present in the most dependent areas (21). Only nine patients (9.9\%) had reactive lymphadenopathy which showed a weak impact on prognosis. Since this is a rare finding in COVID-19 patients, the determination of its role on prognosis needs more validation. We barely could differentiate pleural thickening with trace effusion so we merged these two and considered as trace effusion and found that $62.6 \%$ of patients had a trace or mild pleural effusion which was significantly more in the poor prognosis group. It was consistent with the study conducted by Zhao et al., that found pleural effusion as a helpful feature in the determination of emergency type disease (14). COVID-19 associated architectural distortion and traction bronchiectasis were proposed as poor prognostic factors (14); nevertheless, we could not find any statistically significant correlation. However, we found that the crazy-paving pattern is seen more in the poor prognosis group and could be related to the severity of the disease. On the other hand, we found that the "reversed halo sign" is more common in the good prognosis group, probably indicating remission of injuries. In addition, involvement of central and perivascular areas was not related with poor prognosis. These findings need further evaluations to be validated.

We calculated the CT-score for each lobe and found the mean score of each lung and the total CT-score for each patient. Since only patients with early CT scan findings were enrolled (less than 8 days of symptoms onset), it is the score of early phase disease. The total CT-score was significantly higher in the poor prognosis group. The scoring methods in both previous studies were different from each other, and our study $(13,14)$; however, they also suggested the total CT-score as a prognostic factor in COVID-19 which could help to identify the high-risk patients and give them the appropriate care. These findings imply the need to designate a single, distinct and applicable scoring system. Based on our scoring system we found that 11.5 could be an appropriate cut-off to identify the high-risk patients (sensitivity $67.4 \%$ and specificity $68.7 \%$ ). We also found that the mean score of each lung was correlated with the presence of ipsilateral pleural effusion indicating that pleural effusion happens more in the severely involved lung.

Better performance of the multivariate logistic regression model compared to the univariate model suggests that predicting the prognosis of COVID-19 patients would be more accurate if one considers not only the total CTscore but also other CT scans, demographic and clinical findings which are reasonably expected to have a role in prognosis. We should emphasize though that the power of multivariable logistic models highly depend on the number of cases; therefore, to obtain models with more included inputs, one may need to increase the case number. The finding that the underlying comorbidity did not have a significant contribution to our model might also be the result of the same limitation.

This study had limitations. The number of cases was not sufficient for further analytic studies and enrollment of more factors in predicting the prognosis. We could not perform any follow-up CT scan of the patients. All three hospitals were referral centers for COVID-19 patients, so it is possible that the overall CT-score of the patients in this study would not be representative of the general population. Finally, the clinical and laboratory data of the patients were not complete to be entered into the study and we could not include them in multivariate analysis.

In conclusion, chest CT scan is a valuable imaging method in predicting the prognosis of COVID-19 disease. Among CT findings, the crazy-paving pattern, diffuse distribution, paracardiac and anterior involvement, lymphadenopathy, main pulmonary artery dilation (above 30 $\mathrm{mm}$ ), and pleural effusion were predictors of poor prognosis, while the reversed halo sign was associated with a better prognosis. Multidisciplinary approaches consisting of clinical data, imaging features, and laboratory data would predict the prognosis more accurately.

\section{Acknowledgments}

We thank education development office of Imam Khomeini Hospital Complex for assistance with this project and providing data.

\section{Footnotes}

Authors' Contributions: Study concept and design: M.A.K., B. M., M.C., S.H.; analysis and interpretation of data: P. R., and M.C., S.H.; drafting of the manuscript: M.A.K., B.M., M.C., P.R.; critical revision of the manuscript for important intellectual content: H. G., P. G., and Z. A., H.A.; statistical analysis: P. R.

Conflict of Interests: None declared.

Ethical Approval: IR.TUMS.MEDICINE.REC.1399.095 http://ethics.research.ac.ir/.

Funding/Support: None declared.

Informed Consent: The informed consent was waived by the ethical committee of the Tehran University of medical sciences. 


\section{References}

1. de Groot RJ, Baker SC, Baric RS, Brown CS, Drosten C, Enjuanes L, et al. Middle East respiratory syndrome coronavirus (MERS-CoV): announcement of the Coronavirus Study Group.JVirol.2013;87(14):77902. doi: 10.1128/JVI.01244-13. [PubMed: 23678167]. [PubMed Central: PMC3700179].

2. Drosten C, Gunther S, Preiser W, van der Werf S, Brodt HR, Becker $S$, et al. Identification of a novel coronavirus in patients with severe acute respiratory syndrome. $N$ Engl J Med. 2003;348(20):1967-76. doi: 10.1056/NEJMoa030747. [PubMed: 12690091].

3. Ksiazek TG, Erdman D, Goldsmith CS, Zaki SR, Peret T, Emery S, et al. A novel coronavirus associated with severe acute respiratory syndrome. N Engl J Med. 2003;348(20):1953-66. doi: 10.1056/NEJMoa030781. [PubMed:12690092].

4. Zaki AM, van Boheemen S, Bestebroer TM, Osterhaus AD, Fouchier RA. Isolation of a novel coronavirus from a man with pneumonia in Saudi Arabia. N Engl J Med. 2012;367(19):1814-20. doi: 10.1056/NEJMoa1211721. [PubMed: 23075143].

5. Wang C, Horby PW, Hayden FG, Gao GF. A novel coronavirus outbreak of global health concern. The Lancet. 2020;395(10223):470-3. doi:10.1016/s0140-6736(20)30185-9.

6. Ai T, Yang Z, Hou H, Zhan C, Chen C, Lv W, et al. Correlation of Chest CT and RT-PCR Testing for Coronavirus Disease 2019 (COVID19) in China: A Report of 1014 Cases. Radiology. 2020;296(2):E32-40. doi: 10.1148/radiol.2020200642. [PubMed: 32101510]. [PubMed Central: PMC7233399].

7. Fang Y, Zhang H, Xie J, Lin M, Ying L, Pang P, et al. Sensitivity of Chest CT for COVID-19: Comparison to RT-PCR. Radiology. 2020;296(2):E1157. doi: 10.1148/radiol.2020200432. [PubMed: 32073353]. [PubMed Central: PMC7233365].

8. Huang P, Liu T, Huang L, Liu H, Lei M, Xu W, et al. Use of Chest CT in Combination with Negative RT-PCR Assay for the 2019 Novel Coronavirus but High Clinical Suspicion. Radiology. 2020;295(1):22-3. doi: 10.1148/radiol.2020200330. [PubMed: 32049600]. [PubMed Central: PMC7233360]

9. Xie X, Zhong Z, Zhao W, Zheng C, Wang F, Liu J. Chest CT for typical 2019-nCoV pneumonia: relationship to negative RT-PCR testing. Radiology. 2020:200343.

10. Bernheim A, Mei X, Huang M, Yang Y, Fayad ZA, Zhang N, et al. Chest CT Findings in Coronavirus Disease-19 (COVID-19): Relationship to Duration of Infection. Radiology. 2020;295(3):200463. doi: 10.1148/radiol.2020200463. [PubMed: 32077789]. [PubMed Central: PMC7233369]
11. Pan F, Ye T, Sun P, Gui S, Liang B, Li L, et al. Time Course of Lung Changes at Chest CT during Recovery from Coronavirus Disease 2019 (COVID 19). Radiology. 2020;295(3):715-21. doi: 10.1148/radiol.2020200370. [PubMed: 32053470]. [PubMed Central: PMC7233367].

12. Kanne JP, Little BP, Chung JH, Elicker BM, Ketai LH. Essentials for radiologists on COVID-19: an update-radiology scientific expert panel. Radiological Society of North America; 2020.

13. Yuan M, Yin W, Tao Z, Tan W, Hu Y. Association of radiologic findings with mortality of patients infected with 2019 novel coronavirus in Wuhan, China. PLoS One. 2020;15(3). e0230548. doi 10.1371/journal.pone.0230548. [PubMed: 32191764]. [PubMed Central: PMC7082074].

14. Zhao W, Zhong Z, Xie X, Yu Q, Liu J. Relation Between Chest CT Findings and Clinical Conditions of Coronavirus Disease (COVID-19) Pneumonia: A Multicenter Study. AJR Am J Roentgenol. 2020;214(5):1072-7. doi 10.2214/AJR.20.22976. [PubMed: 32125873].

15. Gong J, Dong H, Xia Q, Huang Z, Wang D, Zhao Y, et al. Correlation analysis between disease severity and inflammation-related parameters in patients with COVID-19 pneumonia. MedRxiv. 2020. doi: 10.1101/2020.02.25.20025643.

16. Wang L, He W, Yu X, Hu D, Bao M, Liu H, et al. Coronavirus disease 2019 in elderly patients: Characteristics and prognostic factors based on 4-week follow-up. J Infect. 2020;80(6):639-45. doi: 10.1016/j.jinf.2020.03.019. [PubMed: 32240670]. [PubMed Central: PMC7118526].

17. Zhou F, Yu T, Du R, Fan G, Liu Y, Liu Z, et al. Clinical course and risk factors for mortality of adult inpatients with COVID-19 in Wuhan, China: a retrospective cohort study. The Lancet. 2020;395(10229):1054-62. doi: 10.1016/s0140-6736(20)30566-3.

18. Mahdavi A, Khalili N, Davarpanah AH, Faghihi T, Mahdavi A, Haseli S, et al. Radiologic Management of COVID-19: Preliminary Experience of the Iranian Society of Radiology COVID-19 Consultant Group (ISRCC). Iranian Journal of Radiology. 2020;17(2). doi: 10.5812/iranjradiol.102324.

19. Chen N, Zhou M, Dong X, Qu J, Gong F, Han Y, et al. Epidemiological and clinical characteristics of 99 cases of 2019 novel coronavirus pneumonia in Wuhan, China: a descriptive study. The Lancet. 2020;395(10223):507-13. doi:10.1016/s0140-6736(20)30211-7.

20. Huang C, Wang Y, Li X, Ren L, Zhao J, Hu Y, et al. Clinical features of patients infected with 2019 novel coronavirus in Wuhan, China. The Lancet. 2020;395(10223):497-506. doi: 10.1016/s0140-6736(20)30183-5.

21. Sheard S, Rao P, Devaraj A. Imaging of acute respiratory distress syndrome. Respir Care. 2012;57(4):607-12. doi: 10.4187/respcare.01731. [PubMed: 22472500]. 